\title{
LncRNA WT1-AS Attenuates hypoxia/ischemia Induced Neuronal Injury in Cerebral Ischemic Stroke via miR-186-5p/XIAP Axis
}

Jianquan You

Jiangsu Taizhou People's Hospital

Fei Qian

Jiangsu Taizhou People's Hospital

Yu Huang

Jiangsu Taizhou People's Hospital

Yingxuan Guo

Jiangsu Taizhou People's Hospital

Yaqian Lv

Jiangsu Taizhou People's Hospital

Yuqi Yang

Jiangsu Taizhou People's Hospital

Xiupan Lu

Jiangsu Taizhou People's Hospital

Ting Guo

Jiangsu Taizhou People's Hospital

Jun Wang ( $\nabla$ wj910188@163.com )

Jiangsu Taizhou People's Hospital https://orcid.org/0000-0002-5511-7914

Bin Gu

Jiangsu Taizhou People's Hospital

\section{Research Article}

Keywords: WT1-AS, Cerebral ischemic stroke, MiR-186-5p, XIAP, Oxygen glucose deprivation

Posted Date: August 20th, 2021

DOI: https://doi.org/10.21203/rs.3.rs-818657/v1

License: (c) (1) This work is licensed under a Creative Commons Attribution 4.0 International License.

Read Full License 


\section{Abstract}

\section{Background}

Cerebral ischemic stroke was a nervous system disease with high occurrence rate and mortality rate. This study aimed to investigate the role and mechanism of IncRNA WT1-AS in cerebral ischemic stroke.

Materials and methods

Starbase and dual luciferase reporter gene assay were used to analyze the target relationship between IncRNA WT1-AS and miR-186-5p. qRT-PCR analysis was used to detect IncRNA WT1-AS and miR-186-5p expression. OGD-induced SH-SY5Y cells injury model was conducted, and cell viability and cell apoptosis were determined by MTT and flow cytometer assay. Caspase3 ability was determined using Caspase3 activity detection kit.

Results

miR-186-5p was a target of IncRNA-WT1-AS. IncRNA WT1-AS was down-regulated and miR-186-5p was up-regulated in blood samples of patients with ischemic stroke and in OGD-induced SH-SY5Y cells. We found that WT1-AS-plasmid promoted OGD-induced cell viability, reduced cell apoptosis and decreased caspase3 ability, and these changes were reversed by miR-186-5p mimic. Subsequently, our results proved that XIAP was a target of miR-186-5p. Similarly, miR-186-5p inhibitor reduced OGD-induced neuronal damage by up-regulating XIAP expression.

Conclusion

IncRNA-WT1-AS/miR-186-5p/XIAP might be a new target for cerebral ischemic stroke treatment.

\section{Introduction}

Stroke is the leading cause of dysfunction and death worldwide [1]. In the United States, the number of strokes approximately reaches 795,000 each year, but more than 140,000 people die from strokes [2]. Strokes are generally divided into three categories, including transient ischemic attack, ischemic stroke and hemorrhagic stroke. $80 \%$ of them are ischemic strokes [3]. The main feature of ischemic stroke is interruption of blood flow, lack of oxygen and glucose in brain cells, which leads to nerve cell function impaired. At present, ischemic stroke has a high morbidity and mortality. The current treatment plan mainly focuses on the following aspects: resolve the infarction in time to limit the continuous damage caused by hypoxic stress and the resulting neuronal damage; and stimulate nerve replacement [4]. Clinically, the use of recombinant tissue plasminogen activator (rt-PA) for intravenous thrombolysis can treat ischemic stroke $[5,6]$. However, the time window of tPA is very short, and there is a risk of hemorrhagic transformation, so the clinical progress is limited [7-9]. So far, the mechanism and treatment of neuronal damage induced by ischemia and hypoxia are still difficult. Recently, more and more studies 
have shown that IncRNA and miRNA play an important role in the mechanism of neuronal damage induced by ischemia and hypoxia.

LncRNA contains about 200 nucleotides. The DNA of the genome is transcribed into RNA, but it cannot be translated into protein [10]. Exercising their biological functions at the RNA level. LncRNA can exert a crucial role in many biological processes, including epigenetic regulation, cell cycle regulation and cell differentiation regulation $[11,12]$. At present, there are more and more reports about IncRNA in various diseases. Many literature reports that the expression of IncRNA is elevated in many diseases, but decreased in some diseases, including cancer, neurological diseases and diabetes [13,14]. WT1-AS is also called Wilms tumor1 antisense RNA, this RNA encodes a zinc finger transcription domain [15]. An increasing evidence showed that WT1-AS is involved in many cancers development, including gastric cancer, non-small cell lung cancer, cervical cancer and glioma [16-19]. Here, we investigated that the role of WT1-AS in cerebral ischemic stroke.

MiRNAs are a general term for a class of small-molecule non-coding RNAs that are about 20 to 22 nucleotides in length, as opposed to mRNA-transcribed proteins. MiRNA did not encode proteins, and inhibited target genes expression [20-22]. Previous research demonstrated that miR-186-5p, as a cancerrelated miRNA, is involved in many cancers occurrence and development [23]. Zhu et al. showed that miR$186-5 p$ that acted as a tumor suppressor gene was negatively expressed in neuroblastoma [24]. Jones et al. showed that miR-186-5p downregulation inhibited cell proliferation, growth and invasion in metastatic cancer cells [25]. Tao et al. indicated that miR-186-5p participated in atherosclerosis development [26]. However, there was no report about the role of miR-186-5p in cerebral ischemic stroke. In our study, through bioinformatics software, we found that there was certain relationship between IncRNA WT1-AS, miR-186-5p and XIAP. Therefore, our aim is to explore the mechanism of three moleculars in cerebral ischemic stroke.

\section{Materials And Methods}

\section{Clinical samples}

30 blood samples of patients with ischemic stroke were collected within 3 hours after the onset of stroke. 30 blood samples of healthy volunteers were collected as the control group. The study procedures were approved by Ethics Committee of Taizhou People's Hospital. All patients agreed in writing to use their specimens in this study.

\section{Dual luciferase reporter analysis}

The wild-type or mutant of WT1-AS was cloned into the pmiRGLO vector (Promega, USA) to verify the relationship between WT1-AS and miR-186-5p. They were co-transfected with miR-186-5p mimic or mimic control and Renilla luciferase into $293 \mathrm{~T}$ cells. After transfection for $48 \mathrm{~h}$, luciferase activity was tested by using Promega kits. 
In order to investigate the reaction of miR-186-5p and XIAP, we constructed WT-XIAP and MUT-XIAP 3'-UTR luciferase reporter gene plasmids. 293T cells were transfected with Renilla luciferase, luciferase reporter gene plasmids and miR-186-5p mimic or mimic control for $48 \mathrm{~h}$. Luciferase activity was tested by using Promega kits according to the instruction.

\section{Cell culture and transfection}

Neuroblastoma cell lines SH-SY5Y cells were acquired from American Tissue Culture Collection (ATCC). The cell line was cultured in DMEM (Gibco, NY, USA) medium with $10 \%$ FBS (Gibco) and cultured at $37^{\circ} \mathrm{C}$ in a $5 \% \mathrm{CO}_{2}$ incubator. SH-SY5Y cells were transfected with WT1-AS-plasmid, control-plasmid, mimic control, miR-186-5p mimic, WT1-AS-plasmid+mimic control, WT1-AS-plasmid+miR-186-5p mimic, inhibitor control, miR-186-5p inhibitor, control-siRNA, XIAP-siRNA, miR-186-5p inhibitor+controlsiRNA, or miR-186-5p inhibitor+XIAP-siRNA using Polyplus reagent transfection reagent (Invitrogen; Thermo Fisher Scientific, Inc.) for 48 hours according to the operation steps. After 48 h, based on transfected cells, we performed following experiments.

\section{qRT-PCR assay}

We obtained total RNA using TRIzol (TaKara) following SOP. Total RNA was transformed into cDNA by reverse transcription using cDNA (Vazyme, Nanjing, Jiangsu). Subsequently, we performed qPCR with SYBR Green PCR kit (Vazyme) followed with the reference manual. GAPDH (for mRNA) or U6 (for miRNA) was used as endogenous control. Gene expression was calculated by $2^{-\Delta \Delta C t}$ formula.

\section{Administration of Oxygen Glucose Deprivation/ Reoxygenation (OGD/R)}

In order to establish an oxygen glucose deprivation/reoxygenation (OGD/R) cell model in vitro, we changed the medium for SH-SY5Y cells and replaced the high-glycemic DMEM medium with the glucosefree Dulbecco's modified Eagle medium. And the cells were grown in a $37{ }^{\circ} \mathrm{C}, 1 \% \mathrm{O}_{2}, 94 \% \mathrm{~N}_{2}$ and $5 \% \mathrm{CO}_{2}$ environment for $4 \mathrm{~h}$; then, we restored the cells to a normal culture state ( $95 \%$ air and $5 \% \mathrm{CO}$ ) and cultured for another $24 \mathrm{~h}$.

\section{Western blot assay}

The cells were lysed using RIPA buffer (Solarbio, Beijing). The protein concentration was acquired by using bicinchoninic acid assay kit (Pierce, Appleton, WI, USA). Equal amount of proteins were isolated by $12 \%$ SDS-PAGE, and then transferred onto PVDF membranes. The membranes were blocked with $5 \%$ non-fat milk to prevent nonspecific binding and then incubated with primary antibodies including anticleaved-caspases $3\left(1: 1000\right.$, abcam) and anti-caspases $3\left(1: 1000\right.$, abcam) at $4^{\circ} \mathrm{C}$ overnight. The next day, they were incubated with secondary antibody for $2 \mathrm{~h}$. The protein signals were visualized by ECL method (Applygen Technologies, Inc). 
Cells apoptosis analysis was performed by using the Annexin-V/propidium iodide (PI) Apoptosis Detection Kit (BD Bioscience). Briefly, after treatment, cells were collected, disposed and centrifuged with low temperature at high speed, and re-suspended in $100 \mu \mathrm{L}$ of FITC-binding buffer. Subsequently, we added approximately $5 \mu \mathrm{L}$ ready-to-use Annexin V-FITC and $5 \mu \mathrm{L}$ PI into the buffer. Cells were incubated for 30 min at room temperature in dark. Annexin V-FITC and PI fluorescence were assessed by BD FACSCalibur flow cytometer (BD Technologies).

\section{MTT assay}

Cells were treated with specific methods and then plated into a 96-well plates and incubated for 24 h. Then, we added $20 \mathrm{~mL}$ MTT ( $5 \mathrm{mg} / \mathrm{ml}$, Sigma) into wells and the cells were further cultured for 4 h. Next, we removed the supernatant and added $200 \mu \mathrm{L}$ dimethyl sulfoxide to the wells. The absorbance were measured at $570 \mathrm{~nm}$.

\section{Caspase3 activity detection}

We detect caspase 3 activity by using a caspase3 activity detection kit (Beyotime Biotechnology). Briefly, we collected transfected cells into a EP tube. The cells were centrifuge at $600 \mathrm{xg}$ for 5 minutes at $4{ }^{\circ} \mathrm{C}$ to collect the cells. We added lysate to the cells in a certain ratio, re-suspended, and lysed for 15 minutes in an ice bath. After centrifuged for 10 minutes, the supernatant was transferred to an ice-bath pre-cooled centrifuge tube. The caspase 3 enzyme activity was measured immediately at $405 \mathrm{~nm}$.

\section{Statistical analysis}

Results were analyzed by GraphPad Prism 6.0 software. Statistical significance of difference between groups was determined by Student's t-test or ANOVA followed by Tukey's post-hoc test. Data was showed as mean $\pm S D$ from three independent experiments. $p<0.05$ was regarded as statistically significant.

\section{Results}

\section{Determination of the binding site of IncRNA WT1-AS and miR-186-5p}

Firstly, we predicted the relationship between WT1-AS and miR-186-5p by bioinformatics tool Starbase. Starbase prediction result showed that miR-186-5p and IncRNA-WT1-AS have mutual binding sites (Figure 1A). Based on software predictions, we found that miR-186-5p is partially complementary to the WT1-AS 3'-untranslated region. Then, 293T cells were co-transfected with WT1-AS-WT, WT1-AS-MUT, miR186-5p mimic or mimic control for $48 \mathrm{~h}$. We performed dual-luciferase reporter assay to detect the activity of luciferase. Dual-luciferase reporter assay indicated that miR-186-5p mimic could inhibit the activity of WT1-AS-WT. However, miR-186-5p mimic could not inhibit the activity of WT1-AS-MUT (Figure 1B). Taken together, miR-186-5p is the target gene of IncRNA-WT1-AS. Next, we collected 30 cases blood samples from ischemic stroke patients within 3 hours after the onset of stroke. qRT-PCR assay results demonstrated that compared with the healthy control group, IncRNA WT1-AS expression was 
substantially down-regulated (Figure 1C), while miR-186-5p expression was up-regulated (Figure 1D) in the blood samples from ischemic stroke patients.

\section{LncRNA WT1-AS was negatively expressed and miR-186-5p was positively expressed in OGD-induced SH- SY5Y cells}

Next, we established OGD-induced SH-SY5Y cell model. LncRNA WT1-AS expression and miR-186-5p expression were detected by qRT-PCR assay. Our results showed that compared with the control group, IncRNA WT1-AS expression was significantly reduced in OGD-induced SH-SY5Y cells (Figure 1E); while miR-186-5p expression was obviously increased (Figure 1F).

\section{WT1-AS negatively regulated miR-186-5p expression in SH-SY5Y cells}

SH-SY5Y cells were plated into a 6-well plate $\left(1 \times 10^{6}\right.$ cells/well $)$ and cultured at $37^{\circ} \mathrm{C}$ for 24 hours. Then WT1-AS-plasmid, miR-186-5p mimic, WT1-AS-plasmid+mimic control or WT1-AS-plasmid+miR-186-5p mimic was transfected into SH-SY5Y cells strictly following the lipofectamine ${ }^{\circledR} 2000$ (Invitrogen; Thermo Fisher Scientific, Inc.) kit instructions. Next we use qRT-PCR analysis to evaluate transfection efficiency. Compared with control-plasmid group, WT1-AS-plasmid increased WT1-AS expression in SH-SY5Y cells (Figure 2A). Compared with mimic control group, miR-186-5p mimic increased miR-186-5p expression (Figure 2B). Compared with control-plasmid group, WT1-AS-plasmid reduced miR-186-5p expression, and this reduction was significantly reversed by miR-186-5p mimic (Figure $2 \mathrm{C}$ ).

\section{LncRNA WT1-AS decreased OGD-induced neuronal damage by reducing miR-186-5p expression}

After cell transfection for 24 hours, SH-SY5Y cells were incubated for 4 hours in serum-free and sugar-free artificial cerebrospinal fluid at $37{ }^{\circ} \mathrm{C}, 1 \% \mathrm{O}_{2}, 94 \% \mathrm{~N}_{2}$, and $5 \% \mathrm{CO}_{2}$, and then replaced DMEM medium to continue to incubate for $24 \mathrm{~h}$ under normoxia. Our experiment is divided into these groups: control group; OGD group; OGD+control-plasmid group; OGD+WT1-AS-plasmid group; OGD+WT1-AS-plas-

mid+mimic control group; OGD+WT1-AS-plasmid+miR-186-5p mimic group. Subsequently, we carried out a series of experiments. Compared with control group, miR-186-5p expression was obviously improved in OGD group; compared with OGD+control-plasmid group, miR-186-5p expression was significantly reduced in OGD+WT1-AS-plasmid group, and the reduction was reversed by miR-186-5p mimic (Figure 3A). qRTPCR analysis showed that IncRNA WT1-AS expression in the OGD group was reduced; compared with the OGD+control-plasmid group, IncRNA WT1-AS expression was increased in the OGD+WT1-AS-plasmid group (Figure 3B). MTT and FCM assay showed that cell viability was decreased (Figure 3C) and cell apoptosis was increased (Figure 3D and E) in the OGD group. Caspase3 activity was increased in the OGD group (Figure 3F). Compared with OGD+control-plasmid group, cell viability was significantly increased (Figure 3C) and cell apoptosis was reduced (Figure 3D and E) in the OGD+WT1-AS-plasmid group, Furthermore, Caspase3 viability was reduced (Figure 3F), and all the effects were reversed by miR186-5p mimic. 


\section{Determination of the relationship between miR-186-5p and XIAP}

Then, through literature analysis and bioinformatics software (TargetScan) analysis, we found that there is a direct binding site between miR-186-5p and XIAP (Figure 4A); the dual luciferase report experiment confirmed the relationship between miR-186-5p and XIAP (Figure 4B). Subsequently, SH-SY5Y cells were seeded into a 6-well plate $\left(1 \times 10^{6}\right.$ cells/well) and cultured at $37^{\circ} \mathrm{C}$ for 24 hours. Inhibitor control, miR-186$5 p$ inhibitor, control-siRNA, XIAP-siRNA, miR-186-5p inhibitor+control-siRNA, or miR-186-5p inhibitor+XIAPsiRNA was transfected into SH-SY5Y cells for $24 \mathrm{~h}$. We performed qRT-PCR assay to assess transfection efficiency. Compared with the inhibitor control group, miR-186-5p inhibitor reduced miR-186-5p expression in SH-SY5Y cells (Figure 4C). Compared with the control-siRNA group, XIAP-siRNA decreased XIAP expression at mRNA level (Figure 4D). Compared with the inhibitor control group, miR-186-5p inhibitor significantly increased XIAP expression at mRNA and protein level. This increase was obviously reversed by XIAP-siRNA transfection (Figure 4E and F). Taken together, XIAP was target gene of miR-186-5p.

\section{Effect of miR-186-5p inhibitor on OGD-induced nerve cell damage}

After cell transfection for $24 \mathrm{~h}, \mathrm{SH}-\mathrm{SY} 5 \mathrm{Y}$ cells were treated as OGD-induced cell injure model. Our experiment is divided into these groups, including OGD+inhibitor control; OGD+miR-186-5p inhibitor; OGD+miR-186-5p inhibitor+control-siRNA; OGD+miR-186-5p inhibitor+XIAP-siRNA. Compared with the control group, miR-186-5p was up-regulated (Figure 5A) and XIAP mRNA (Figure 5B) was reduced in OGD group; compared with the OGD+inhibitor control group, miR-186-5p was down-regulated and XIAP mRNA was increased in OGD+miR-186-5p inhibitor group. Compared with the OGD+inhibitor control group, cell viability was improved in OGD+miR-186-5p inhibitor group (Figure 5C), cell apoptosis was reduced (Figure $5 \mathrm{D}$ and $\mathrm{E}$ ), Caspase3 viability (Figure 5F), and these effects were reversed by XIAP-siRNA.

\section{Discussion}

Acute cerebral ischemia stroke leads to higher mortality in humans, and also causes some complications, such as behavioral, social, attention, cognitive and functional motor dysfunction $[27,28]$. The pathological process of ischemic stroke is more complicated, and the contributing factors are diverse. Refers to previous literature description, neuroinflammation and oxidative stress are important factors of ischemic brain injury $[29,30]$. Although there have been many treatments in the past period of time, including thrombolytic therapy, anticoagulation therapy, carotid endarterectomy, and neuroprotective agents, there are still many problems with current treatment programs [31-33]. Therefore, we need to explore new approach to treat cerebral ischemic stroke.

LncRNA has now attracted more people's attention in various diseases including cerebral ischemic stroke. Shan et al. showed that IncRNA TUG1 participated in cerebral ischemic stroke development, and TUG1 down-regulation reduced OGD/R-induced damage [34]. A report from Wen's team indicated that IncRNA Gm4419 up-regulation promoted TNF-a, IL-1 $\beta$ and IL-6 expression, thereby increasing OGD/R damage [35]. Hu et al. showed that knocking down IncRNA SNHG15 could improve ischemia/hypoxia-induced 
neuronal damage and microglia inflammation by targeting miR-302a-3p/STAT1 [36]. Xiao et al. suggested that IncRNA H19 was highly expressed in ischemic stroke patients and H19 siRNA decreased neuronal apoptosis in OGD neuronal cells through trageting miR-19a [37]. In our experiment, we researched the role of IncRNA WT1-AS in cerebral ischemic stroke. Firstly, we employed bioinformatics analysis and dual luciferase reporter analysis to show that there was binding site between IncRNA WT1AS and miR-186-5p.

Previous research demonstrated that IncRNA WT1-AS was involved in cancer development. WT1-AS expression was decreased in cervical carcinoma, papillary thyroid carcinoma (PTC) and cervical squamous cell carcinoma (CSCC). In addition, Cui et al. showed that WT1-AS overexpression suppressed cell viability by miR-330-5p/p53 axis in cervical carcinoma [38]. Le et al. reported that WT1-AS inhibited $\mathrm{IHH}-4$ cell proliferation through miR-203 and survivin in PTC [39]. Similarly, WT1-AS suppressed CSCC cell proliferation by p53 in Zhang's team research [40]. However, there was basically no report on the study of IncRNA WT1-AS in ischemic stroke. Same as the previous result, our results showed that WT1-AS expression was reduced in blood sample of patients, while miR-186-5p expression was increased. Consistent with the expression in the patient, WT1-AS was up-regulated and miR-186-5p was downregulated in OGD-induced SH-SY5Y cells in vitro.

Next, we explored the action mechanism of WT1-AS and miR-186-5p in OGD-induced SH-SY5Y cell injure. Du et al. showed that LINC01705 is targeted by miR-186-5p and LINC01705 participated in BC development by miR-186-5p [41]. Zhu et al. reported that HCP5 promoted neuroblastoma cell proliferation by down-regulated miR-186-5p [42]. In osteosarcoma, miR-186-5p suppressed cell proliferation, invasion and metastasis by targeting FOXK1 [43]. In our research, WT1-AS overexpression decreased miR-186-5p expression in SH-SY5Y cells and WT1-AS reduced OGD-induced neuronal damage by reducing miR-186$5 p$ expression. Subsequently, we found that XIAP was target of miR-186-5p.

XIAP, X-linked inhibitor of apoptosis protein, is an important member of the apoptotic protein (IAP) family and is a cytoplasmic inhibitor of caspases $-3,-7$ and -9 [44,45]. As a target for cancer treatment, XIAP was abnormally expressed in cancer and played an important role in regulating the resistance to death [46]. Zhang et al. indicated that miR-130a promoted cisplatin resistance of cells in ovarian cancer through regulating XIAP [47]. A report from Deng's team demonstrated that XIAP participated in ischaemic stroke development and XIAP expression was decreased in MCAO rats. XIAP siRNA reversed the effect suppressed miR-130a on neurological function and angiogenesis in MCAO rats. In this study, miR-186-5p inhibitor alleviated OGD-induced SH-SY5Y cells injure. These findings indicated that WT1-AS exerted a crucial role in cerebral ischemic stroke through miR-186-5p-XIAP axis.

In conclusion, IncRNA WT1-AS reduced neuronal damage caused by hypoxia/ischemia in cerebral ischemic stroke through miR-186-5p/XIAP axis.

\section{Declarations}

\section{Funding}


This study was supported by Scientifc Research Foundation of Taizhou People's Hospital (ZL202015) and the Project of Taizhou City Personnel Bureau (RCPY201937).

\section{Competing interests}

The authors declare that they have no competing interests.

\section{Availability of data and material}

The datasets used and/or analyzed during the present study are available from the corresponding author on reasonable request.

\section{Code availability}

Not applicable.

\section{Ethics approval}

The study procedures were approved by Ethics Committee of Taizhou People's Hospital. All patients agreed in writing to use their specimens in this study.

\section{Consent to participate}

All patients agreed in writing to use their specimens in this study.

\section{Consent for publication}

All patients agreed for publication.

\section{Acknowledgments}

Not applicable.

\section{References}

1. Meschia JF, Bushnell C, Boden-Albala B, et al (2014) Guidelines for the primary prevention of stroke. Stroke 45,3754-3832. https://doi: 10.1161/STR.0000000000000046.

2. Virani SS, Alonso A, Benjamin EJ, et al (2020) Heart disease and stroke statistics-2020 update: a report from the american heart association. Circulation 141:e139-e596. https://doi: 10.1161/CIR.0000000000000757.

3. Thrift AG, Dewey HM, Macdonell RA, McNeil JJ, Donnan GA (2001) Incidence of the major stroke subtypes: initial findings from the North East Melbourne stroke incidence study (NEMESIS). Stroke 32:1732-1738. https://doi: 10.1161/01.str.32.8.1732. 
4. George PM, Steinberg GK (2015) Novel Stroke Therapeutics: unraveling stroke pathophysiology and its impact on clinical treatments. Neuron 87:297-309. https://doi: 10.1016/j.neuron.2015.05.041.

5. Fjetland L, Kurz KD, Roy S, Kurz MW (2015) Evaluation of the recombinant tissue plasminogen activator pretreatment in acute stroke patients with large vessel occlusions treated with the direct bridging approach. Is it worth the effort? Eur J Neur 22:322-327. https://doi: 10.1111/ene.12569.

6. Hommel M (1996) Tissue plasminogen activator for acute ischemic stroke. N Engl J Med 334:14051406.

7. Wardlaw JM, Murray V, Berge E, del Zoppo G, Sandercock P, Lindley RL, Cohen G (2012) Recombinant tissue plasminogen activator for acute ischaemic stroke: an update systematic review and metaanalysis. Lancet 379:2364-2372. https://doi: 10.1016/S0140-6736(12)60738-7.

8. Whiteley WN, Slot KB, Fernandes P, Sandercock P, Wardlaw J (2012) Risk factors for intracranial hemorrhage in acute ischemic stroke patients treated with recombinant tissue plasminogen activator: a systemic review and meta-analysis of 55 studies. Stroke 43:2904-2909. https://doi: 10.1161/STROKEAHA.112.665331.

9. Yaghi S, Eisenberger A, Willey JZ (2014) Symptomatic intracerebral hemorrhage in acute ischemic stroke after thrombolysis with intravenous recombinant tissue plasminogen activator: a review of natural history and treatment. JAMA Neurol 71:1181-1185. https://doi:

10.1001/jamaneurol.2014.1210.

10. Kung JT, Colognori D, Lee JT (2013) Long noncoding RNAs: Past, present, and future. Genetics 193:651-669. https://doi: 10.1534/genetics.112.146704.

11. Lekka E, Hall J (2018) Noncoding RNAs in disease. FEBS Lett 592:2884-900. https://doi: 10.1002/1873-3468.13182.

12. Kopp F, Mendell JT (2018) Functional classification and experimental dissection of long noncoding RNAs. Cell 172:393-407. https://doi: 10.1016/j.cell.2018.01.011.

13. Carter G, Miladinovic B, Patel AA, Deland L, Mastorides S, Patel NA (2015) Circulating long noncoding RNA GAS5 levels are correlated to prevalence of type 2 diabetes mellitus. BBA Clin 4:102-107. https://doi: 10.1016/j.bbacli.2015.09.001. e

14. Zheng Y, Song D, Xiao K, et al (2016) LncRNA GAS5 contributes to lymphatic metastasis in colorectal cancer. Oncotarget 7:83727-83734. https://doi: 10.18632/oncotarget.13384.

15. Lv L, Chen G, Zhou J, Li J, Gong J (2015) WT1-AS promotes cell apoptosis in hepatocellular carcinoma through down-regulating of WT1. J Exp Clin Cancer Res 34:119. https://doi: 10.1186/s13046-015-0233-7.

16. Yin YW, Du L, Li XZ, et al (2018) LncRNA WT1-AS/miR-494-3p Regulates Cell Proliferation, Apoptosis, Migration and Invasion via PTEN/PI3K/AKT Signaling Pathway in Non-Small Cell Lung Cancer. OncoTargets and Therapy. J Cell Physiol 2018;1-14. https://doi: 10.2147/OTT.S278233.

17. Du T, Zhang B, Zhang S, et al (2016) Decreased expression of long non-coding RNA WT1-AS promotes cell proliferation and invasion in gastric cancer. Biochim Biophys Acta 1862:12-19. https://doi: 10.1016/j.bbadis.2015.10.001. 
18. Qiu G, Tong W, Jiang C, et al (2020) Long Noncoding RNA WT1-AS Inhibit Cell Malignancy via miR494-3p in Glioma. Technol Cancer Res Treat 19:1533033820919759. doi:

$10.1177 / 1533033820919759$.

19. Luo H, Zhang J, He Z, Wu S (2021) Long Noncoding RNA WT1-AS Inhibits the Progression of Cervical Cancer by Sponging miR-205. Cancer Biother Radiopharm 36:491-500. https://doi: 10.1089/cbr.2019.3279.

20. Seungil R, Chanjae P, David Y, Kenton M S, Yan W (2007) Tissue-dependent paired expression of miRNAs. Nucleic Acids Res 35:5944-5953. https://doi: 10.1093/nar/gkm641.

21. Mallory AC, Vaucheret $\mathrm{H}$ (2004) MicroRNAs: something important between the genes. Curr Opin Plant Biol 7:120-125. https://doi: 10.1016/j.pbi.2004.01.006.

22. Garzon R, Calin GA, Croce CM (2009) MicroRNAs in cancer. Annu Rev Med 60:167-179. https://doi: 10.1146/annurev.med.59.053006.104707.

23. Esquela-Kerscher A, Slack FJ (2006) Oncomirs-microRNAs with a role in cancer. Nat Rev Cancer 2006;6:259-269. https://doi: 10.1038/nrc1840.

24. Zhu K, Su Y, Xu B, et al (2019) MicroRNA-186-5p represses neuroblastoma cell growth via downregulation of Eg5. Am J Transl Res 11:2245-2256.

25. Jones DZ, Schmidt ML, Suman S, et al (2018) Micro-RNA-186-5p inhibition attenuates proliferation, anchorage independent growth and invasion in metastatic prostate cancer cells. BMC Cancer 18:421. https://doi: 10.1186/s12885-018-4258-0.

26. Tao Z, Cao Z, Wang X, Pan D, Jia Q (2021) Long noncoding RNA SNHG14 regulates ox-LDL-induced atherosclerosis cell proliferation and apoptosis by targeting miR-186-5p/WIPF2 axis. Hum Exp Toxicol 40:47-59. https://doi: 10.1177/0960327120940363.

27. Ren C, Li N, Gao C, et al (2020) Ligustilide provides neuroprotection by promoting angiogenesis after cerebral ischemia. Neurol Res 42:683-692. https://doi: 10.1080/01616412.2020.1782122.

28. Li B, Concepcion K, Meng X, Zhang L (2017) Brain-immune interactions in perinatal hypoxic-ischemic brain injury. Prog Neurobiol 159:50-68. https://doi: 10.1016/j.pneurobio.2017.10.006.

29. Tobin MK, Bonds JA, Minshall RD, Pelligrino DA, Testai FD, Lazarov $O$ (2014) Neurogenesis and inflammation after ischemic stroke: what is known and where we go from here. J Cereb Blood Flow Metab 34:1573-84. https://doi: 10.1038/jcbfm.2014.130.

30. Yu W, Gao D, Jin W, Liu S, Qi S (2018) Propofol prevents oxidative stress by decreasing the ischemic accumulation of succinate in focalcerebral ischemia-reperfusion injury. Neurochem Res 43:420-429. https://doi: 10.1007/s11064-017-2437-z.

31. Deb P, Sharma S, Hassan KM (2010) Pathophysiologic mechanisms of acute ischemic stroke: An overview with emphasis on therapeutic significance beyond thrombolysis. Pathophysiology 17:197218. https://doi: 10.1016/j.pathophys.2009.12.001.

32. Liu LP, Wong LK, Wang DZ, Miao ZR (2014) Current status of endovascular procedures in management of ischemic stroke in china. CNS Neurosci Ther 20:483-484. https://doi: $10.1111 /$ cns.12281. 
33. Ebinger M, Winter B, Wendt M, et al (2014) Effect of the use of ambulance-based thrombolysis on time to thrombolysis in acute ischemic stroke: a randomized clinical trial. JAMA. 2014 Apr 2330;311(16):1622-31. https://doi: 10.1001/jama.2014.2850.

34. Shan W, Chen W, Zhao X, et al (2020) Long noncoding RNA TUG1 contributes to cerebral ischaemia/reperfusion injury by sponging mir-145 to up-regulate AQP4 expression. J Cell Mol Med 24:250-259. https://doi: 10.1111/jcmm.14712.

35. Wen Y, Yu Y, Fu X (2017) LncRNA Gm4419 contributes to OGD/R injury of cerebral microglial cells via IKB phosphorylation and NF-KB activation. Biochem Biophys Res Commun 487:923-929. https://doi: 10.1016/j.bbrc.2017.05.005.

36. Hu CT, Li C, Ma QY, et al (2021) Inhibition of long noncoding RNA SNHG15 ameliorates hypoxia/ischemia-induced neuronal damage by regulating miR-302a-3p/STAT1/NF-kB axis. Yonsei Med J 62:325-337. https://doi: 10.3349/ymj.2021.62.4.325.

37. Xiao Z, Qiu Y, Lin Y, et al (2019) Blocking IncRNA H19-miR-19a-Id2 axis attenuates hypoxia/ischemia induced neuronal injury. Aging (Albany NY) 11:3585-3600. https://doi: 10.18632/aging.101999.

38. Cui L, Nai M, Zhang K, Li L, Li R (2019) LncRNA WT1-AS inhibits the aggressiveness of cervical cancer cell via regulating p53 expression via sponging miR-330-5p. Cancer Manag Res 11:651-667. https://doi: 10.2147/CMAR.S176525. e

39. Li F, Luo P, Ouyang Q, Zhong XM (2020) LncRNA WT1-AS downregulates survivin by upregulating miR-203 in papillary thyroid carcinoma. Cancer Management and Research 2020;12:443-449. https://doi: 10.2147/CMAR.S232294.

40. Zhang $Y, N a ~ R$, Wang X (2019) LncRNA WT1-AS up-regulates $p 53$ to inhibit the proliferation of cervical squamous carcinoma cells. BMC Cancer 19:1052. https://doi: 10.1186/s12885-019-6264-2.

41. Du C, Zhang JL, Wang Y, et al (2020) The Long Non-coding RNA LINC01705 Regulates the Development of Breast Cancer by Sponging miR-186-5p to Mediate TPR Expression as a Competitive Endogenous RNA. Front Genet 11:799. https://doi: 10.3389/fgene.2020.00779.

42. Zhu K, Wang L, Zhang X, et al (2021) LncRNA HCP5 promotes neuroblastoma proliferation by regulating miR-186-5p/MAP3K2 signal axis. J Pediatr Surg 56:778-787. https://doi: 10.1016/j.jpedsurg.2020.10.011.

43. Zhang Z, Zhang W, Mao J, et al (2019) miR-186-5p functions as a tumor suppressor in human osteosarcoma by targeting FOXK1. Cell Physiol Biochem 52:553-564. https://doi: 10.33594/000000039.

44. Dasari VR, Velpula KK, Kaur K, et al (2010) Cord blood stem cell-mediated induction of apoptosis in glioma downregulates X-linked inhibitor of apoptosis protein (XIAP). PLoS One 5:e11813. https://doi: 10.1371/journal.pone.0011813.

45. Rehm M, Huber HJ, Dussmann $H$, et al (2006) Systems analysis of effector caspase activation and its control by X-linked inhibitor of apoptosis protein. EMBO J 25:4338-4349. https://doi: 10.1038/sj.emboj.7601295. 
46. Obexer P, Ausserlechner MJ (2014) X-linked inhibitor of apoptosis protein-a critical death resistance regulator and therapeutic target for personalized cancer therapy. Front Oncol 4:197. https://doi: 10.3389/fonc.2014.00197.

47. Zhang X, Huang L, Zhao Y, et al (2013) Downregulation of miR-130a contributes to cisplatin resistance in ovarian cancer cells by targeting X-linked inhibitor of apoptosis (XIAP) directly. Acta Biochim Biophys Sin 2013;45:995-1001. https://doi: 10.1093/abbs/gmt113.

\section{Figures}

A

Target: 5' uuccgucuuagACAAUUCUUUC 3'

|| ||||||||

MIRNA : 3' ucggguuUuCCUCUUAAGAAAC 5'

C

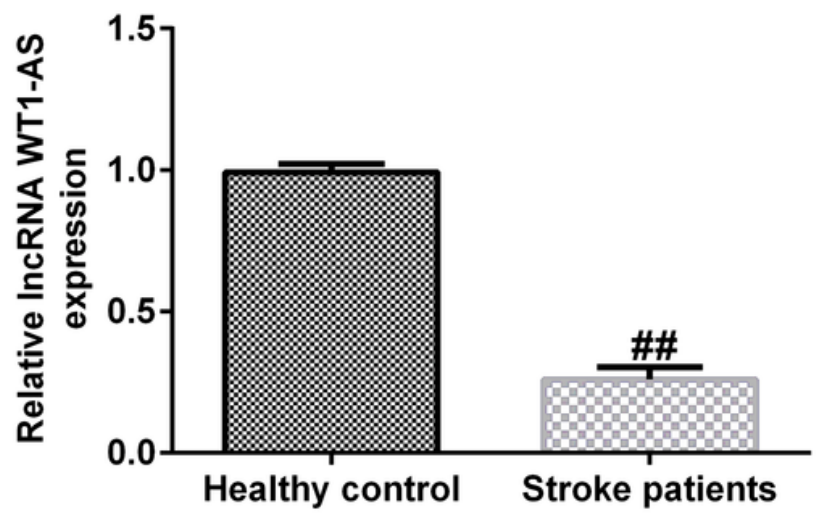

E

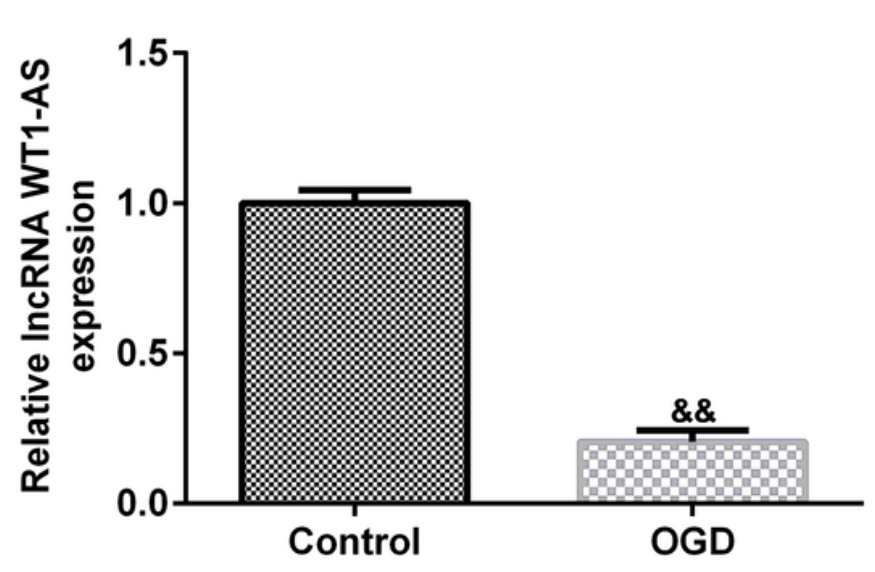

B

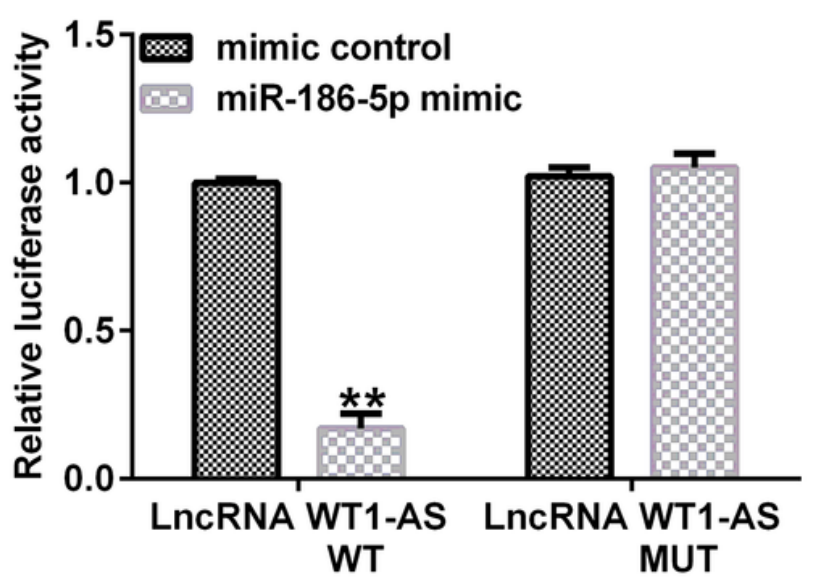

D

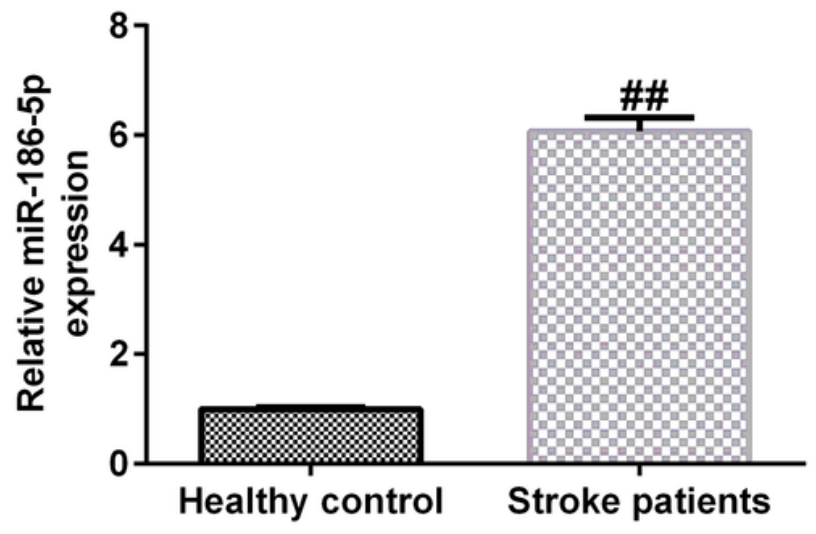

$\mathbf{F}$

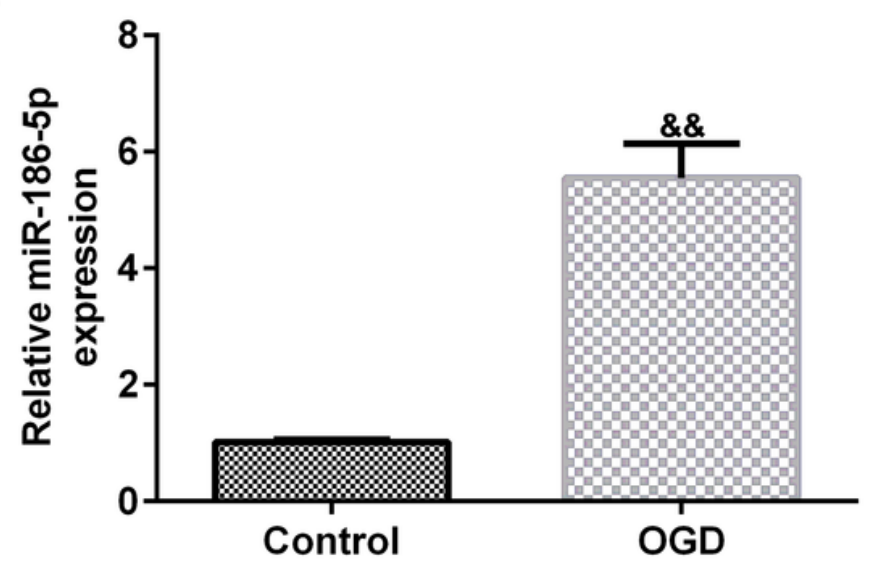




\section{Figure 1}

The expression levels of IncRNA WT1-AS and miR-186-5p in ischemic stroke patients and OGD-induced SH-SY5Y cells. (A). Interaction between miR-186-5p and 3'UTR of WT1-AS was predicted using Starbase prediction software. (B). Dual luciferase reporter gene assay that verification of the interaction between WT1-AS and miR-186-5p when 293T cells were co-transfected with miR-186-5p mimic and wild-type or mutant WT1-AS 3'-UTR reporter. (C and D) Expression of WT1-AS and miR-186-5p in the blood samples from ischemic stroke patients and healthy control. (E). qRT-PCR analysis detected IncRNA WT1-AS expression at mRNA level. (F). qRT-PCR analysis detected miR-186-5p expression at mRNA level. ${ }^{\star \star} p<0.01$ vs. mimic control; \#\#p<0.01 vs. Healthy control; \&\&p<0.01 vs. Control.
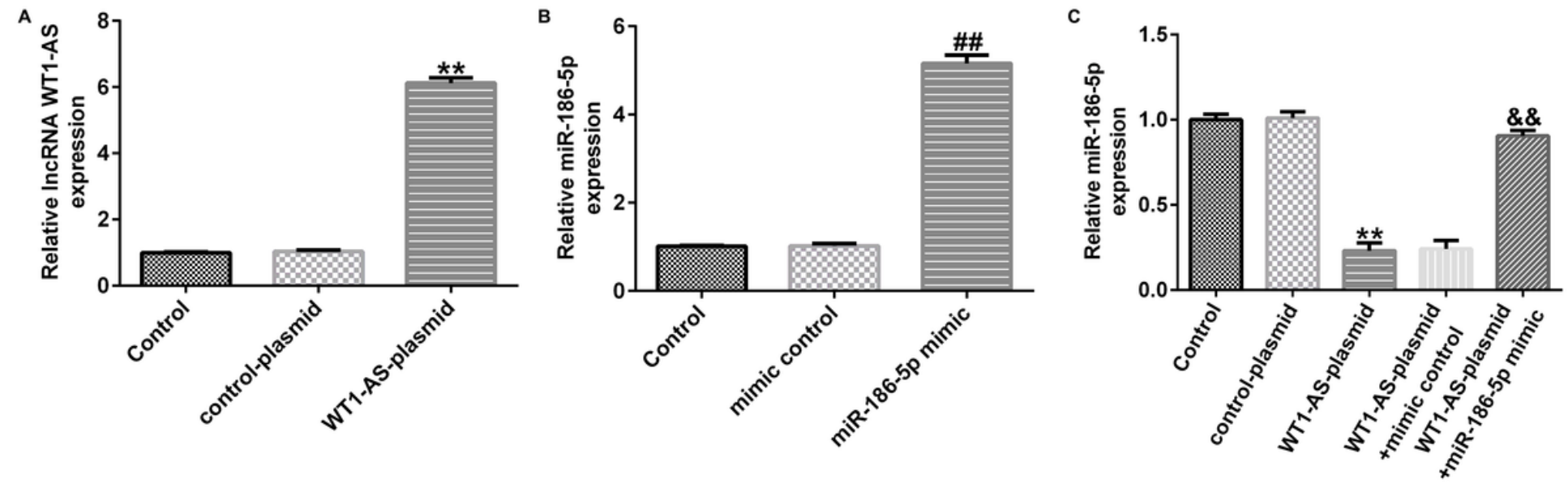

\section{Figure 2}

IncRNA WT1-AS negatively regulated miR-186-5p expression in SH-SY5Y cells. (A). qRT-PCR analysist of WT1-AS expression when SH-SY5Y cells were transfected with control-plasmid or WT1-AS-plasmid. (B). qRT-PCR analysis of miR-186-5p expression when SH-SY5Y cells were transfected with mimic control or miR-186-5p mimic. (C). qRT-PCR analysis that miR-186-5p expression when SH-SY5Y cells were transfected with control-plasmid, miR-186-5p mimic, WT1-AS-plasmid+mimic control or WT1-ASplasmid+miR-186-5p mimic. ${ }^{* *} p<0.01$ vs. Control-plasmid; \#\#p<0.01 vs. Mimic control; \&\&p<0.01 vs. WT1-AS-plasmid+mimic control. 

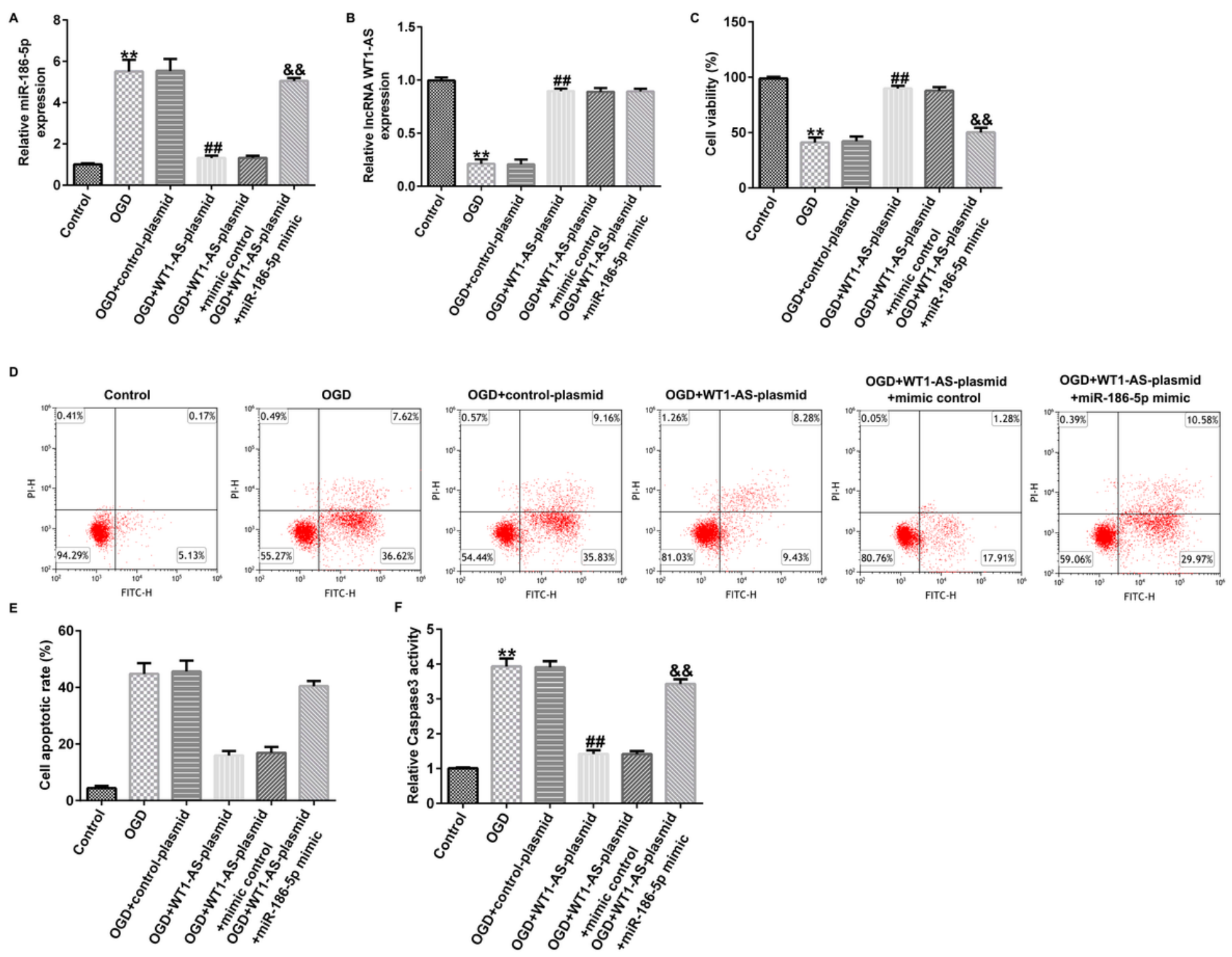

Figure 3

The effect of LncRNA WT1-AS on nerve cell injury induced by OGD. (A). qRT-PCR analysis of miR-186-5p expression. (B). qRT-PCR analysis of IncRNA WT1-AS expression. (C). MTT assay of cell viability. (D). FCM assay of cell apoptosis. (E). The apoptosis ratio were calculated and presented. (F). The caspase3 activity was detected by specific kit. ${ }^{* \star p}<<0.01$ vs. Control; $\# \# p<0.01$ vs. $0 G D+m i m i c$ control; \&\&p<0.01 vs. OGD+WT1-AS-plasmid+mimic control. 
A

Position 332-338 of XIAP $3^{\prime}$ UTR 5

hsa-miR-186-5p

.. UCAUCUGGAUUUUUUATUCTUUU. . UCGGGUUUUCCUCUUAGGAAC

IIIIIII

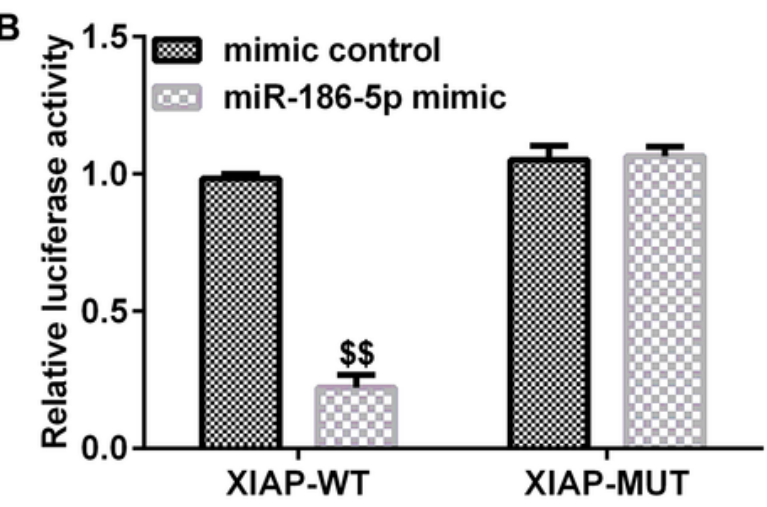

C

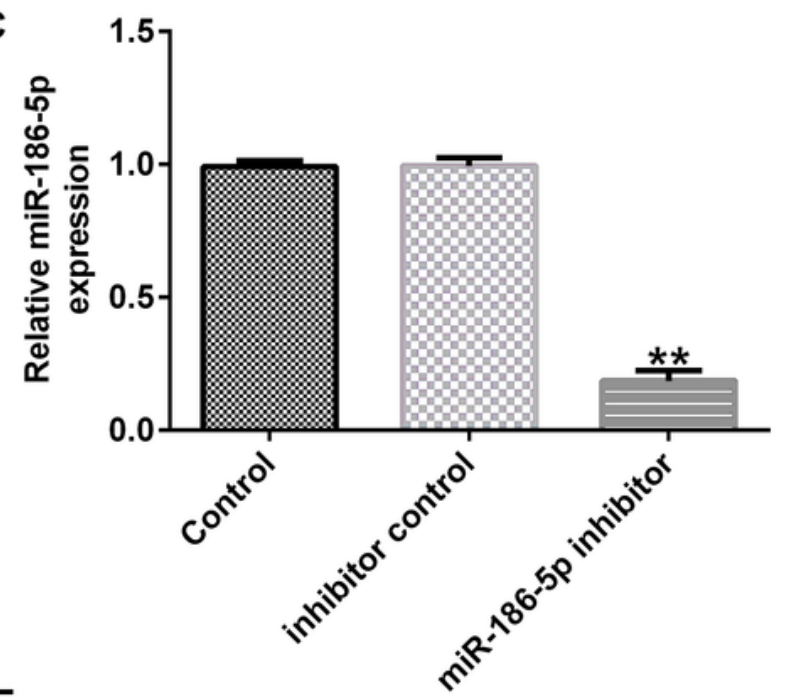

$\mathbf{E}$

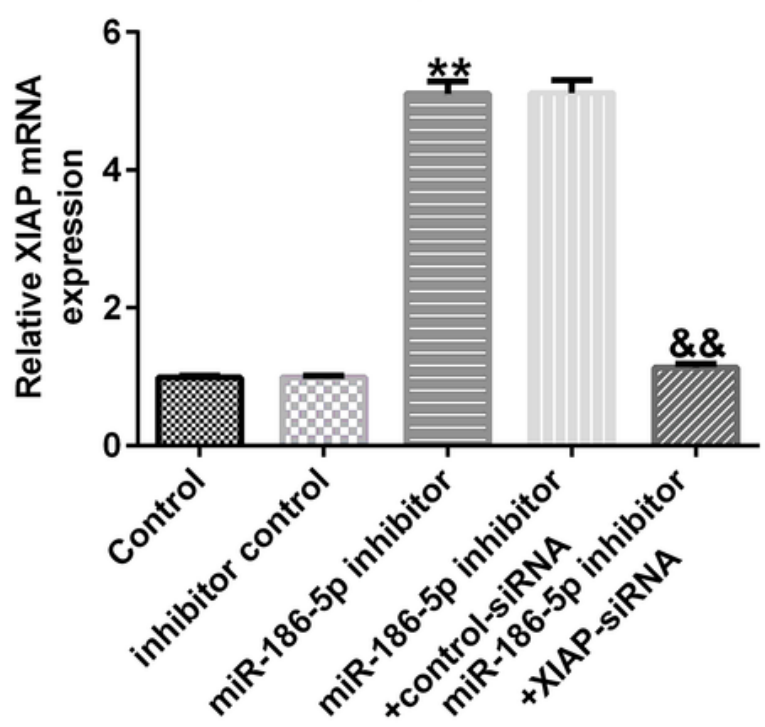

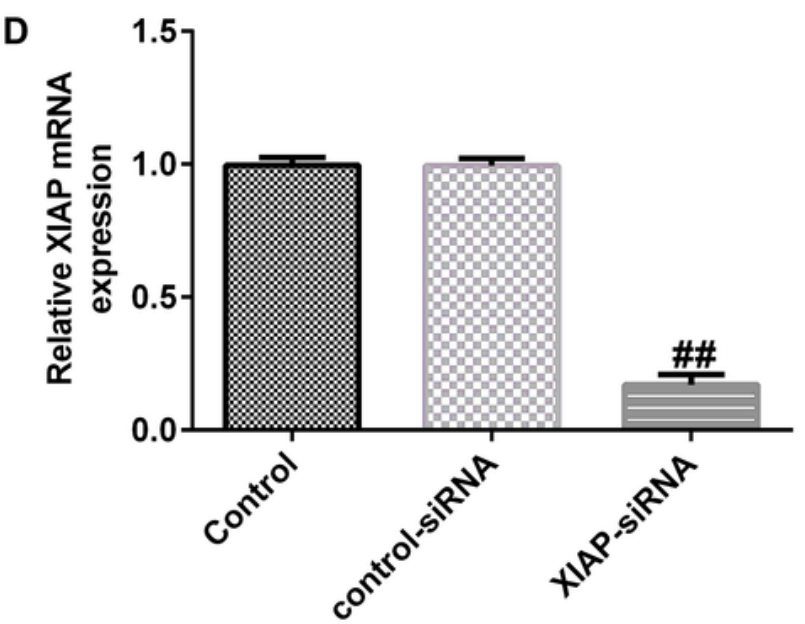

$\mathbf{F}$

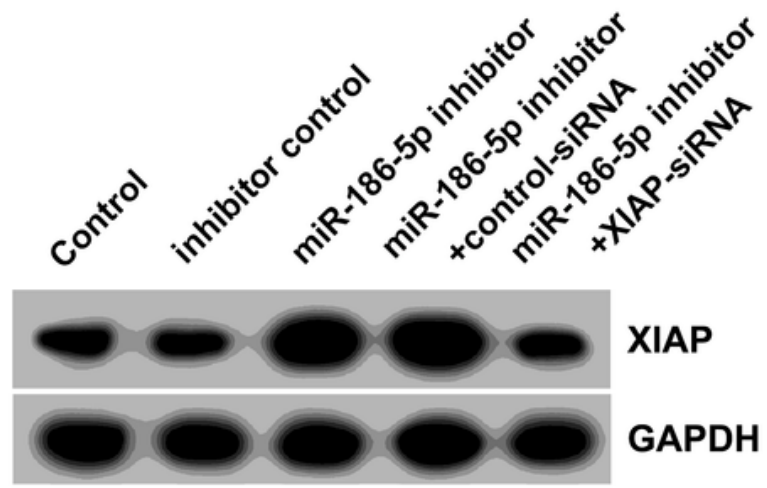

Figure 4

XIAP was target of miR-186-5p and miR-186-5p negatively regulated XIAP expression in SH-SY5Y cells. (A). Interaction between miR-186-5p and 3'UTR of XIAP was predicted using TargetScan. (B). Dual luciferase reporter gene assay that verification of the interaction between miR-186-5p and XIAP when 293T cells were co-transfected with miR-186-5p mimic and wild-type or mutant XIAP 3'-UTR reporter. (C). qRT-PCR assay of miR-186-5p expression in SH-SY5Y cells transfected with inhibitor control or miR-186- 
$5 p$ inhibitor. (D). qRT-PCR assay of XIAP expression in SH-SY5Y cells transfected with control-siRNA or XIAP-siRNA. (E). qRT-PCR assay of XIAP expression in SH-SY5Y cells transfected with miR-186-5p inhibitor+control-siRNA, or miR-186-5p inhibitor+XIAP-siRNA. (F). Western blot analysis of XIAP expression at protein level. $\$ \$ p<0.01$ vs. mimic control; $* \star p<0.01$ vs. Inhibitor control; $\# \# p<0.01$ vs. Control-siRNA; \&\&p<0.01 vs. miR-186-5p inhibitor+control-siRNA.
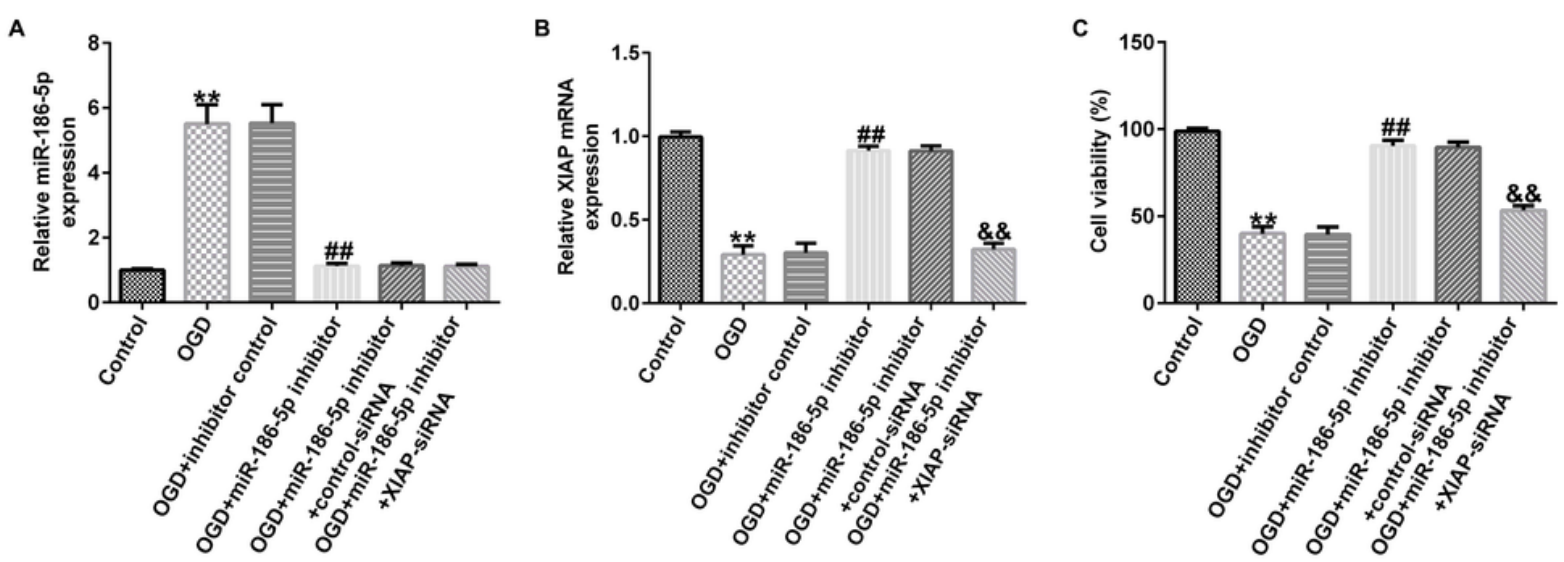

D
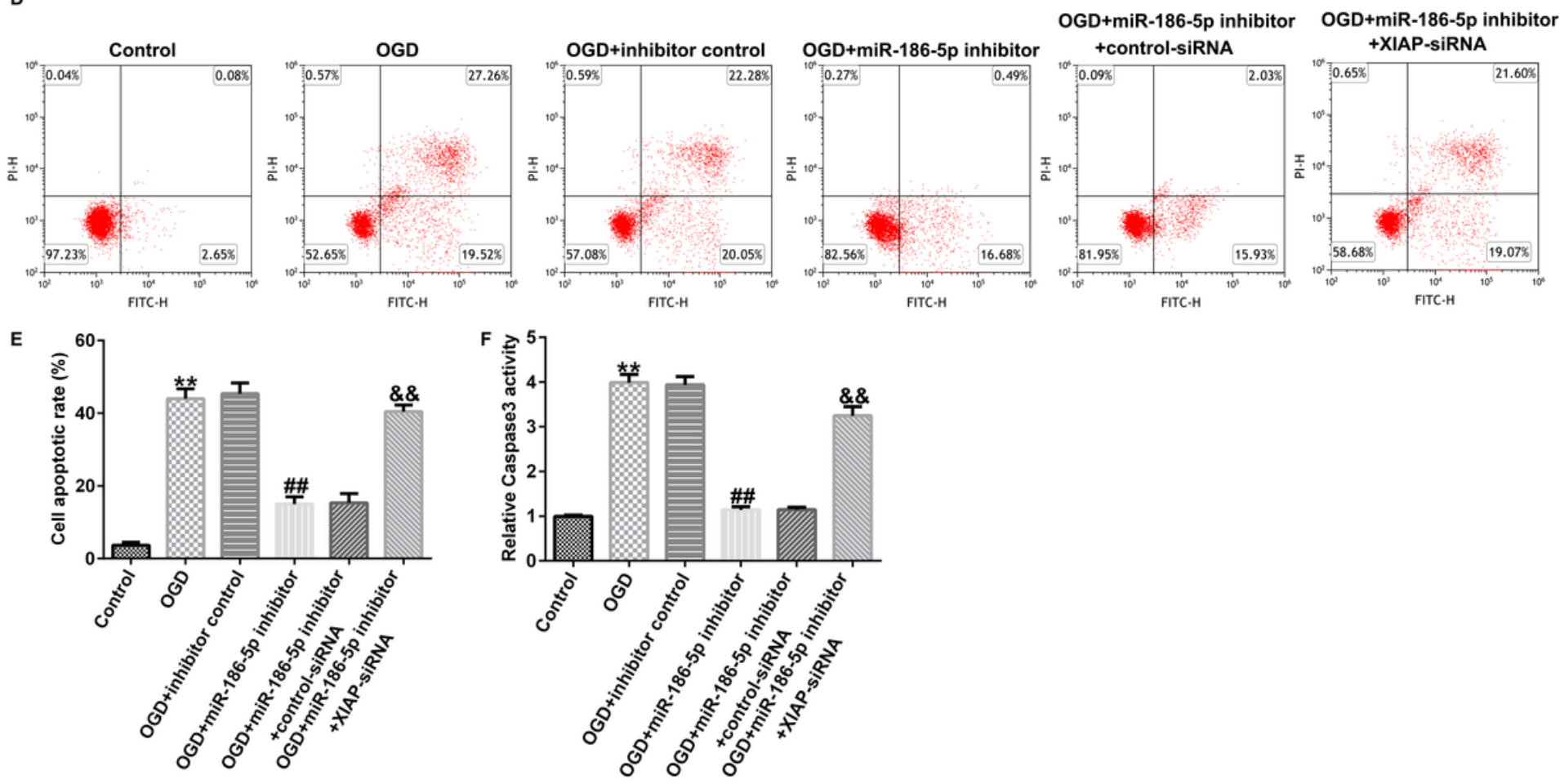

Figure 5

MiR-186-5p inhibitor reduced OGD-induced neuronal damage by up-regulating XIAP expression. (A). qRTPCR analysis of miR-186-5p expression. (B). qRT-PCR analysis of XIAP expression. (C). MTT assay detected cell viability. (D). FCM assay determined cell apoptosis. (E). The apoptosis ratio were calculated and showed. (F). The Caspase3 activity was detected by specific kit. ${ }^{* \star} p<0.01$ vs. Control; $\# \# p<0.01$ vs. OGD+inhibitor control; \&\&p<0.01 vs. OGD+miR-186-5p inhibitor+control-siRNA. 